\title{
Prevalencia de autoanticuerpos en pacientes con falla reproductiva
}

\author{
J. Cubillos; A. Lucena; C. Lucena; J.C. Mendoza; J. Ferro; H. Ruiz; A. Arango; G. Quiroga; E. Lucena*
}

RESUMEN: En este estudio se investiga la incidencia de autoanticuerpos en pacientes que no han tenido ningún embarazo a término.

Se seleccionaron 43 pacientes con infertilidad primaria y 110 con antecedentes de pérdida del embarazo. En el primer grupo se encontró una incidencia de $37.2 \%(P<0.05)$ para anticuerpos antinucleares (ANA) y $53.5 \%$ para anticuerpos antifosfolípidos IG G e IG M (APL) $(\mathbf{P}<\mathbf{0 . 0 0 5})$.

Para el grupo de pacientes con antecedentes de aborto, el $31.8 \% \quad(\mathrm{P}<0.05)$ fue positivo para anticuerpos antinucleares y $38.2 \%$ para anticuerpos antifosfolípidos $(\mathbf{P}<0.05)$.

Como grupo control se utilizaron 35 pacientes sanas con fertilidad comprobada y sin ningún antecedente de pérdida del embarazo ni de enfermedad autoinmune, para este grupo la incidencia fue de 5.7\% para ANA y $11.4 \%$ para APL.

La alta incidencia de autoanticuerpos observada en pacientes con infertilidad primaria sugiere la implicación directa de estos anticuerpos en la falla reproductiva y consecuentemente en procedimiento de IVF y reproducción asistida.

Se ha descrito ampliamente la prevalencia de ANA y APL en pacientes con antecedentes de pérdida recurrente del embarazo (PRE), en este estudio observamos la presencia de autoanticuerpos aún después del primer aborto.

Podemos entonces concluir que las pacientes con algún antecedente de falla reproductiva deben ser inmunológicamente evaluadas y tratadas antes de ser sometidas a técnicas de fertilización asistida o a un nuevo embarazo en el caso de las pacientes con PRE.

PALABRAS CLAVES: Antinucleares, antifosfolípidos, infertilidad primaria, falla reproductiva.

SUMMARY: The purpose of these study was determine the incidence of autoantibodies in patients with no term pregnancies.

Patients selected included 43 with primary infertility and 110 with a history of pregnancy loss. In the first group the incidence of antinuclear antibodies (ANA) and IG G and IG M antiphospholipid (APL) was 37.2\% (P<0.05) and 53.5\% (P<0.05) respectively.

In the group of patients with history of miscarriage, $31.8 \%(P<0.05)$ were positive for antinuclear antibodies and $38.2 \%(P<0.05)$ for antiphospholipid antibodies.

Controls were 35 healthy patients with proven fertility and no history of pregnancy loss or autoimmune disease. In this group incidence was $5.7 \%$ for ANA and $11.4 \%$ for APL.

The high incidence of autoantibodies foun in patients with primary infertility might suggest a direct involvement of these antibodies in reproductive failure and consequently in IVF and assisted fertility procedures.

The prevalence of ANA and APL has been extensively described in patients with history of recurrent pregnancy losses (RPL). In this study we observed the appearance of antibodies even after the first miscarriage.

We concluded that patients with a history of reproductive failure should immunologically evaluated and treated before undergoing assisted fertilization techniques or before a new pregnancy in those cases of RPL.

KEY WORDS: Antinuclear antibodies, antiphospholipids, primary infertility, reproductive failure.

\section{Introducción}

El tratamiento de la infertilidad mediante la Reproducción asistida y la transferencia de embriones ha sido practicada y desarrollada con éxito por casi 20 años.

Centro Colombiano de Fertilidad y Esterilidad CECOLFES. División de Inmunología en Biomedicina Reproductiva Bogotá, Colombia.
(Edwards y Craft, 1978). A pesar de los avances tecnológicos las tasas de implantación por transferencia de embriones no se han incrementado significativamente y oscilan entre el 10 y $15 \%$. (Society of Assited Reproductive Technology, The American Fertility Society, 1993). Las bajas tasas de implantación y embarazo han estimulado estudios para identificar los factores que podrían afectar los mecanismos de implantación y el posterior desarrollo placentario. 
Los aspectos inmunológicos han sido considerados como uno de los posibles factores para la falla reproductiva, ya que participan directamente en el proceso incluyendo la fertilización, implantación y placentación.

La fertilización e implantación representan un reconocimiento inmunológico alogénico necesario para el éxito del desarrollo del transplante gestacional. Aunque los mecanismos no son claros, se conoce que el embrión en desarrollo emite señales que inducen actividades inmunomoduladoras, antiluteolíticas o luteotrópicas necesarias para aumentar la receptividad del endometrio y facilitar la implantación.

Hay especulación que procesos autoinmunes similares a aquellos asociados con la pérdida recurrente del embarazo podrían comprometer la implantación del preembrión. (Sher, et al 1994).

Los anticuerpos reducen la eficacia de la implantación y promueven el rechazo autoinmune del concepto. Se han propuesto diversos mecanismos que podrían explicar este tipo de falla reproductiva.

Aunque la fisiopatología de los anticuerpos antinucleares en reproducción permanecen sin esclarecer, respuestas autoinmunes al DNA y a las Historias relacionados con título bajo de ANA y un patrón de fluorescencia moteado, se asocian con procesos inflamatorios a nivel del tejido placentario con su consecuente repercusión a nivel fetal. (Beer et al 1993).

Los APL han sido relacionados con una variedad de manifestaciones clínicas, eventos tromboembólicos, trombocitopenia y complicaciones obstétricas (Triplett et al, 1988). PRE, Preeclampsia de iniciación temprana, Corea Gravidarum, Retardo de Crecimiento Intrauterino y muerte fetal han sido patologías observadas con mayor frecuencia en pacientes con anticuerpos antifosfolípidos. (Triplett et al, 1989).

La placenta es un órgano muy sensible a la acción de los Anticuerpos Antifosfolípidos (APL) los cuales pueden bloquear la respuesta aloinmune al impedir que los fosfolípidos se unan al receptor de Interleuquina 2 del Linfocito $\mathrm{T}$, desencadenando la activación de la inmunidad citotóxica, lo que ocasiona la destrucción del trofoblasto y la consecuente pérdida fetal. (Beer et al, 1994).

Los fosfolípidos actúan como moléculas de adhesión en la formación del sincitiotrofoblasto a partir de citotrofoblasto. Los acs contra estas moléculas ocasionan un daño en el desarrollo del sincitiotrofoblasto y por lo tanto la pérdida fetal.

Los APL han sido implicados en el daño endotelial y alteración en la cascada de la coagulación que ocasionan fenómenos trombóticos tanto vasculares como venosos. (Feinstein et al 1985).

Las lesiones ocasionadas a nivel placentario conllevan a fallas en el aporte nutricional del concepto (insuficiencia placentaria) lo que se relaciona con problemas de Aborto Recurrente, Retardo de Crecimiento Intrauterino y posterior muerte fetal. (Carreras et al 1981, Lubbe et al 1982, Branch et al 1984, Lubbe et al 1985, McNeil et al 1990, Lockwood et al 1994).
El propósito de este estudio es investigar la incidencia de autoanticuerpos en pacientes que asisten a CECOLFES y en quienes está indicada la Fertilización In Vitro o algún procedimiento de Reproducción Asistida y tratamiento de la PRE.

\section{Materiales y métodos}

\section{Pacientes}

Se incluyeron 153 pacientes que asistieron a CECOLFES desde el primero de Octubre de 1995 hasta Abril 30 de 1996. La selección de las pacientes fue randomizada, independiente de la raza y de la edad. Todas ellas fueron evaluadas para las etiologías de infertilidad más comunes, enfermedades infecciosas (Clamidia HIV, Hepatitis B, etc.), perfil endocrinológico (Estradiol, FSH, y LH en día 3 del ciclo, PRL), laparoscopia e histeroscopia y ultrasonido transvaginal para estudios de ovulación. Para la valoración del factor masculino, un examen físico, espermograma computarizado y niveles de PRL.

Las pacientes fueron clasificadas según su historia clínica: 43 con infertilidad primaria sin diferenciar su etiología y 110 con historia de pérdida recurrente del embarazo. (Una o más).

El segundo grupo a su vez se dividió así: 34 pacientes con 1 aborto (Al), 47 pacientes con 2 pérdidas (A2), 19 con 3 (A3) y 10 pacientes con 4 o más $(A>4)$. Y como grupo control 35 pacientes no embarazadas sin ningún antecedente de pérdida del embarazo o enfermedad autoinmune y con fertilidad comprobada. (Ruiz et al 1995).

\section{Evaluación del Laboratorio}

\section{Valoración Autoinmune}

Anticuerpos antinucleares. Se determinaron por la técnica de Inmunofluorescencia Indirecta, reactivo comercial de Kallestad Quantafluor, utilizando como sustrato células de epitelio humano HEP-2. Títulos iguales o superiores a 1/40 fueron considerados como positivos. Para la determinación del Título final se hicieron diluciones seriadas de la muestra.

Anticoagulante Lúpico. Para su investigación se utilizaron tres pruébas, la primera mediante la determinación del APTT (cephaloplastina Baxter), considerándose positiva si existe una prolongación igual o mayor a 6 segundos con relación a un control normal. El índice de Tromboplastina Diluida donde se establece una relación con el control normal y se considera positivo un índice mayor o igual a 1.3 y la tercera prueba es la Neutralización de Plaquetas que indica si el inhibidor de la coagulación es dependiente de los fosfolípidos. La positividad en mínimo dos de estas pruebas identifica la presencia del anticoagulante lúpico. (Kelsy et al., 1984, Alvin et al., 1987).

Para la identificación de anticuerpos Antifosfolípidos se utilizó un microelisa específico para cada antígeno, Cardiolipina, Fosfatidiletanolamina; Fosfatidilserina, Fosfatidilglicerol, Acido Fosfatídico y Fosfatidilinositol, tanto para Ig G como para Ig M. (Harris et al 1983, Loizou 
et al 1985). Las placas de poliestileno se fijaron con el respectivo antígeno (SIGMA), se lavaron con PBS (Buffer Fosfato Salino) y posteriormente se bloquearon con una solución de PBS-Suero Bovino Fetal al $10 \%$ por dos horas a temperatura ambiente. El Suero de las pacientes y de los controles diluidos $1 / 50$ en PBS-SBF 10\%, fueron agregados a las placas e incubados por dos horas a temperatura ambiente. Después de lavar las placas tres veces con PBS, se agregaron $50 \mu \mathrm{l}$ de conjugado anti Ig G o IGM humano, marcado con Fosfatasa Alcalina. Después de una hora de incubación, las placas fueron lavadas 5 veces con PBS, y se les agregó $50 \mu \mathrm{l}$ de $\mathrm{P}$ nitrofenil Fosfato, sustrato cromogénico, y después de aproximadamente $15 \mathrm{~min}$ a $37^{\circ} \mathrm{C}$ se frenó la reacción con NAOH $3 \mathrm{M}$. Luego se determinó la densidad óptica (O.D) a $405 \mathrm{~nm}$. Los valores para los puntos de corte, fueron obtenidos de controles normales, considerándose como valores positivos las absorbancias por encima de 2 Desviaciones Stándar de la Media y como positivos altos los valores por encima de la Media más 5 Desviaciones Stándar.

\section{Análisis estadístico}

Para la comparación entre los diversos grupos y el grupo control se utilizó Chi cuadrado aplicando la corrección de Yates de acuerdo con las características de los grupos. El análisis de las edades se realizó mediante la t de Student. El análisis estadístico fue realizado con el programa Statistics PC DOS versión 2.0.

\section{Resultados}

La Tabla 1 muestra la distribución de edades en los diferentes grupos y en el grupo control.

La incidencia de anticuerpos antinucleares en el grupo de pacientes con infertilidad primaria fue de $31.2 \%$ (16/43) comparado con 5.7\% (2/35) del grupo control $(\mathrm{p}<0.01)$.

En el grupo de pacientes con antecedentes de abortos se observó positividad en el $31.8 \%$ (35/110). Siendo significativo para los grupos de abortadoras $(\mathrm{p}<0.050)$. (fig. 1).

Figura 1

INCIDENCIA ANA

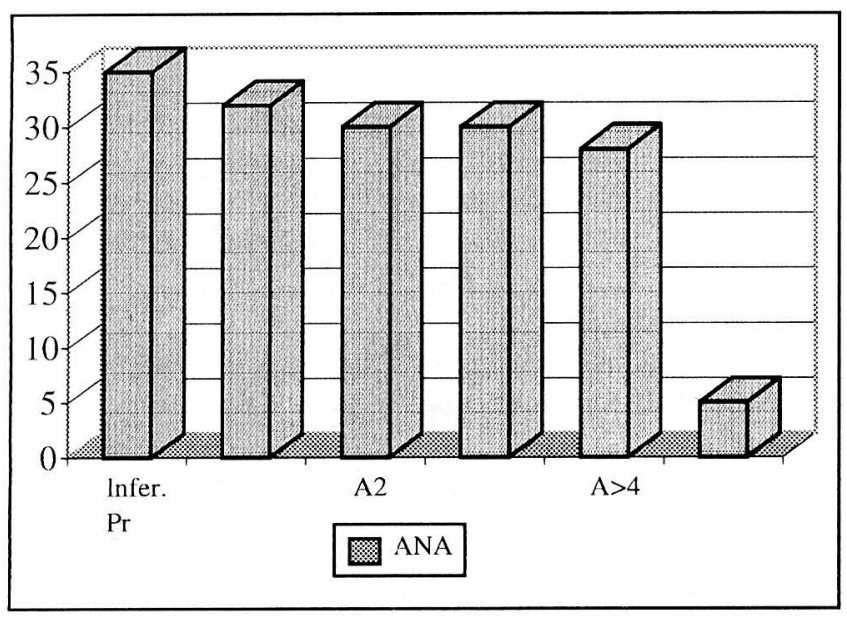

Tabla 1

EDAD DE PACIENTES Y GRUPO CONTROL

\begin{tabular}{|llll|}
\hline & $\begin{array}{l}\text { Inf. } \\
\text { Primaria }\end{array}$ & Abortad. & Control \\
\hline & $\mathrm{n}=43$ & $\mathrm{n}=110$ & $\mathrm{n}=35$ \\
Edad & $33.3 \pm 3.7$ & $34.0 \pm 5.2$ & $24.8 \pm 3.3$ \\
$\mathrm{t}$ & $\mathrm{NS}$ & $\mathrm{NS}$ & \\
\hline
\end{tabular}

NS, diferencias no significativas por la t student.

En pacientes con infertilidad primaria (grupo 1), se observó una incidencia de APL de 53.5\% (23/43) comparado con un $11 \%(4 / 35)$ del grupo control $(\mathrm{P}<0.005)$. En el grupo de abortadoras (grupo 2), 38.2\% (42/110). (p<0.05) (fig. 2) y (fig.3).

En el grupo 1, para los anticuerpos anticardiolipina (ACA), se encontró un $46.5 \%$ (20/43), un $26.3 \%$ (29/110) en el grupo 2 y un $2.9 \%(1 / 35)$ en el grupo control.

Figura 2

INCIDENCIA ANTIFOSFOLIPIDOS

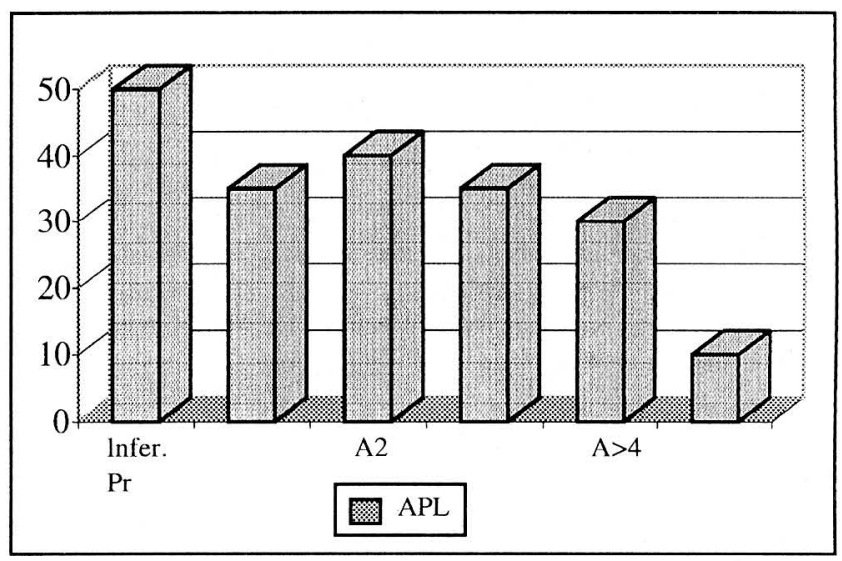

Figura 3

INCIDENCIA APL EN GRUPOS

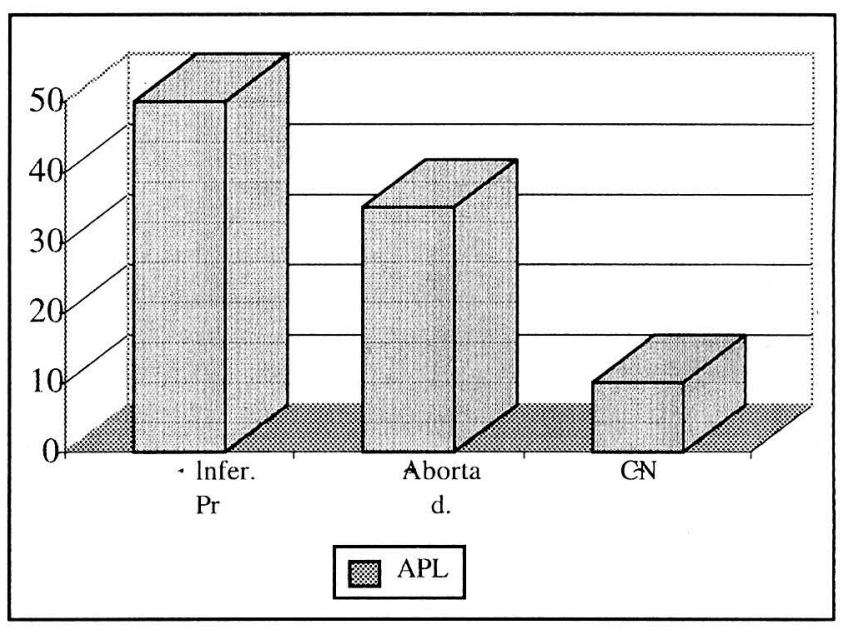


Tabla 2

INCIDENCIA DE ANTICUERPOS ANTIFOSFOLIPIDOS

\begin{tabular}{|llllll|}
\hline & $\begin{array}{l}\text { Infer } \\
\text { Primar. }\end{array}$ & Abort. & Control & $\begin{array}{l}\text { Infe } \\
\text { Prim. }\end{array}$ & Abor \\
\hline Cardiolipina & $20 / 46.5 \%$ & $29 / 26.4 \%$ & $1 / 2.9 \%$ & $<0.005$ & $<0.005$ \\
F. Etanolamina & $11 / 23.9 \%$ & $25 / 22.7 \%$ & $3 / 8.6 \%$ & $<0.050$ & $<0.05$ \\
F. Serina & $14 / 32.6 \%$ & $29 / 26.4 \%$ & $2 / 5.7 \%$ & $<0.050$ & $<0.050$ \\
F. Glicerol & $12 / 27.9 \%$ & $28 / 25.5 \%$ & $4 / 11.4 \%$ & $\mathrm{NS}$ & $\mathrm{NS}$ \\
AC. Fosfatídico & $9 / 20.9 \%$ & $26 / 23.6 \%$ & $2 / 5.7 \%$ & $\mathrm{NS}$ & $<0.050$ \\
F. Inositol & $11 / 23.9 \%$ & $28 / 25.4 \%$ & $2 / 5.7 \%$ & $<0.050$ & $<0.050$ \\
N & 43 & 110 & 35 & $\mathrm{P}$ & $\mathrm{P}$ \\
\hline
\end{tabular}

Para la incidencia de acs contra la fosfatidiletanolamina un $23.9 \%$ para grupo $1,22.7 \%$ para grupo 2 y $8.6 \%$ para el grupo control. Acs. contra fosfatidilserina, $32.6 \%$ (14/43) grupo 1, 26.4\% (29/110) grupo 2 y $5.7 \%$ (2/35) grupo control.

Acs. contra el fosfatidilglicerol, $27.9 \%(12 / 43)$ en infertilidad primaria, $25.5 \%(28 / 110)$ en pacientes con antecedente de aborto y $11.4 \%$ (4/35) en el grupo control. Acs. contra el Acido Fosfatídico y el Fosfatidilinositol en el grupo $1,20.9 \%(9 / 43)$ y $23.9 \%(11 / 43)$ respectivamente, en el grupo $2,23.6 \%(26 / 110)$ y $25.4 \%(28 / 110)$ y en el grupo control 5.7\% (2/35) para ambos epitopes (tabla 2).

En solo dos pacientes que correspondían al grupo A1 se observó positividad para el anticoagulante lúpico.

Estos resultados demuestran una alta incidencia de anticuerpos antinucleares y antifosfolípidos en pacientes con falla reproductiva con relación al grupo control.

Aunque su implicación directa que la infertilidad y en la PRE aún permanece en estudio, es un factor que debe ser tomado en consideración en el estudio de estas pacientes.

\section{Discusión}

El obstetra realiza análisis de rutina en pacientes fértiles, grupo de más alto riesgo para el inicio de una enfermedad autoinmune, y sin embargo es la entidad menos investigada y diagnosticada. Estudios secuenciales muestran que los estrógenos regulen la síntesis de inmunoglobulinas lo cual a su vez incrementa la producción de autoantígenos que podrían estimular el desarrollo de la patología autoinmune. (Grossman, 1984).

Los hallazgos de este estudio en el grupo de pacientes con antecedentes de pérdida del embarazo corrobora reportes previos sobre la asociación de autoanticuerpos y aborto habitual. (Cowchock, et al 1986, Gleicher, et al 1989, Kwak et al 1992, Espinel et al, 1994, Ruiz et al 1995). Sinembargo en este estudio encontramos cambios serológicos aún en pacientes con antecedente de una pérdida.

Aunque los mecanismos que estimulan o inducen la producción de estos autoanticuerpos permanecen sin esclarecer, se sabe que muerte del tejido fetal dentro del útero induce la manifestación de antígenos feto-placentarios que promueven respuestas inmunológicas maternas.

Entre este grupo de autoanticuerpos, los APL, han sido los más estudiados y relacionados con la PRE
(Lockwood et al, 1986, Feinstein et al 1985, Lubbe et al 1985, Scott et al 1987, Beer et al 1993).

En reproducción, los fosfolípidos actúan como moléculas de adhesión en el desarrollo del sincitiotrofoblasto a partir del citotrofoblasto; actúan en la formación de mioblastos que a su vez forman miotúbulos, intervienen en la formación de fibroblastos para la configuración de bandas sincitiales. El trofoblasto los utiliza uniendo la membrana celular con heparan sulfato para contribuir al desarrollo placentario. Alrededor de la semana 12 de gestación, el trofoblasto sincitial libera un lípido de bajo peso molecular cuya función es estabilizar la membrana plasmática de los linfocitos $\mathrm{T}$ ayudadores, bloqueando la expresión de Interleukina 2 y la activación de la proteinkinasa C, controlando la inmunidad mediada por células. Por lo anterior los fosfolípidos estimularían una respuesta aloinmune que favorece el inmunotropismo. (Beer et al. 1993).

Respecto a la coagulación los fosfolípidos actúan en tres niveles, los fosfolípidos de membrana son liberados con la activación de la vía extrínseca y los plaquetarios a su vez con la activación de la vía intrínseca. Además se requiere de fosfolípidos para la conversión de Protrombina a Trombina. (Khamashta et al, 1988).

A partir de los fosfolípidos presentes en las células endoteliales se produce ácido araquidónico, precursor de la prostaciclina, sustancia vasodilatadora y antiagregante plaquetario. De la misma manera, en las plaquetas se producen tromboxanos, que tienen acción vasoconstrictora y favorecen la agregación plaquetaria. Del equilibrio entre tromboxanos y prostaciclina depende parte de la hemostasis sanguínea.

El diagnóstico definitivo de APL depende en gran parte del conocimiento de su etiología y de su clínica. Los APLS parecen causar más daño vascular a través de los mecanismos trombóticos que de la vasculitis. Existen hipótesis acerca de los mecanismos fisiopatológicos de estos acs, ellos incluyen activación plaquetaria, daño en células endoteliales, inhibición de la síntesis de prostaciclina, reducción en la actividad fibrinolítica, bloqueo de la respuesta normal del embarazo, y activación de la inmunidad local citotóxica. (Feinstein et al, 1985) Los APL representan un modelo de patología materno-fetal. Se ha demostrado su implicación en la homeostasis materna con su consecuente repercusión en el desarrollo y crecimiento fetal. 
En este estudio se investigó la incidencia de autoanticuerpos en pacientes con infertilidad primaria que a pesar de los diversos tratamientos no han conseguido el embarazo. La alta incidencia de Acs crea la necesidad de investigar más a fondo este interesante grupo de pacientes y relacionar si la producción de los Acs es inherente al posible factor de la infertilidad (endometriosis, enfermedad pélvica inflamatoria, adhesiones, etc). (Sher et al. 1994), si es una manifestación transitoria producida durante la estimulación de la ovulación (Fish et al, 1991) o si es una consecuencia de la manipulación mecánica en el estudio y tratamiento de estas pacientes.

La prevalencia de APL en la población general se ha reportado entre 5-17\%, (Ruiz et al, 1995). En este estudio reportamos un $53.5 \%$ en las pacientes con infertilidad primaria, esto nos hace relacionar la contribución de estos Acs y la inhabilidad de concebir naturalmente o mediante técnicas de Reproducción asistida.

Los factores inmunológicos participan de una manera directa en todo el proceso reproductivo, implantación, placentación y posterior desarrollo fetal. Por lo tanto los resultados de este estudio respaldan las hipótesis de la implicación directa de la patología autoinmune no sólo en la pérdida recurrente del embarazo sino en la infertilidad primaria.

Estos hallazgos nos hacen pensar que la patología inmunológica está presente en un alto porcentaje en pacientes con falla reproductiva, por lo tanto estos análisis deben continuarse para hacer diagnóstico, tratamiento y futuras evaluaciones.

\section{BIBLIOGRAFIA}

1. Geva, E., Amit, A., Lemer-Geva, L., Azem, F., Yovel, I, and Lessing, J.B. Autoimmune disorders: another posible cause for in-vitro fertilization and embryo transfer failure. Hum. Reprod., 1995; 10 : 2560-2563.

2. Sher, G., Feinman, M., Zouves, C., Kuttner, G. Maassarani, G, et al. High Fecundity rates following in-vitro fertilization and embryo transfer in antiphopholipid antibody seropositive women treated with heparin and aspirin. Hum. Reprod. 1994; 9: 2278-2283.

3. Coulam, C., Krysa L., and Bustillo, M. Intravenous immunoglobulin for in-vitro fertilization failure. Hum. Reprod. 1994; 9: 2265-2269.

4. Gleicher, N., Pratt, D., Dudkiewicz, A. What do we really know about antibody abnormalities and reproductive failure: A critical review. Autoimmunity., 1993; 16: 115-140.

5. Espinel, E., Mendoza, J., Cubillos, J., Botero, E. Autoinmunidad en pacientes con pérdida recurrente del embarazo. Revista Colombiana de Obstetricia y Ginecología. 1994; 45: 109-114.

6. Kwak, J., Beer, A., Cubillos, J., Sandoval, P., Mendoza, J, and Espinel, F. Biological basis of fetoplacental antigenic determinants in the induction of the antiphospholipid antibody syndrome and recurrent pregnancy loss. Annals of the New York Academy of Sciences. 1994; 731: 242-245.

7. Kwak, J., Gilman-Sachs, A., Beaman, K, and Beer, A. Autoantibodies in women with primary recurrent spontaneous abortion ofunknown etiology. J. Reprod Immunol 1992; 22: 15-31.

8. Ruiz, J., Cubillos, J., Mendoza, J., Espinel, F., Kwak, J, and Beer, A. Autoantibodies to phospholipids and nuclear antigens in non-pregnant and pregnant Colombian women with recurrent spontaneous abortions. J. Reprod. Immunol 1995; 28: 41-51.

9. Hams, E., Gharavi, A., Boey, M., Patel, B., Maackworth-Young, C, and Loizou, S. Anticardiolipin antibodies: detection by radioimmunoassay and association with thrombosis in systemic lupus erythematosus. Lancet 1993; 2: 1211-1214.

10. Hams, E.N., Gharavi, A.E, and Hughes, G.R. Antiphospholipid antibodies. Clin. Rheum. Dis., 1985; 11: 591-609.

11. Billington, R.E. From transplantation biology $t$ reproductive immunobiology. Obstet. Gynecol. 1978; 7: 1-14.

12. Beer, A.E., Kwak, Y.H., Sachs, A.G, and Beaman, K.D. New horizons in the evaluation and treatment of the recurrent pregnancy loss. Immunobiology of Reproduction. Serono Symposia, USA, 1994; 316-334.

13. Triplett, D.A., Brandt, J.T. Lupus Anticoagulants: misnomer, paradox, riddle, epiphenomenon. Hematol. Pathol. 1988; 2: 121.

14. Triplett, D.A. Antiphospholipid antibodies and recurrent pregnancy loss. Am J Reprod Immunol Microbiol. 1989; $20: 52$.

15. Loizou, S., Mccrea, J.D., Rudqe, A.C., Reynolds, R. Boyle, C.C., Harris, E.N. Measurement of anticardiolpin antibodies by an enzyme linked immunosorbent assay (ELISA): Standarization and quantitation of results. Clin Exp Immunol. 1985; 62: 738.

16. Feinstein, D.I., Rapaport, S.I. Acquired inhibitors ofblood coagulation. Prog Hemost Thromb. 1972; 1: 75.

17. Feinstein, D.I. Lupus anticoagulant, thrombosis and fetal loss. NEngl J Med. 1985; 313: 1348.
18. Carreras, L.O., Machin, S.J., Deman, R. Arterial thrombosis, intrauterine death and lupus anticoagulant: detection of immunoglobulin interfering with prostacydin formation Lancet, 1981; 1: 244.

19. Lubbe, W.F., Butler, W.S., Palmer, S.J., Liggins, G.C. Lupus anticoagulant in pregnancy. Br J Obstet Gynaecol. 1984; 91: 357.

20. Lubbe, W.F., Pattison, N., Liggins, G.C. Antiphospholipid antibodies and pregnancy. N Engl J Med. 1985; 313: 1350.

21. Lockwood, C.J, and Rand, J.H. The immunobiology and obstetrical consequences of antiphospholipid antibodies. Obstet Gynecol Surg. 1994; 49, 432-441.

22. Kelsy, P.R., Stevenson, K.J., Poller, L. The diagnosis of lupus anticoagulants by the activated partial thromboplastin time- the central role of phosphatidyl serine. Thromb Haemost. 1984; 52: 172.

23. Alvin, B.M., Barr, C.F. The dilute phospholipid APTT: evaluation of specificity for antibodies against cardiolipin and phosphatidyl serine. Thromb Haemost. 1987; 58: 391.

24. Coulam, C.B., Mclntyre, J.A., lvagenknecht, D., Faulk, W.P., Rote, N.S. Interlaboratory inconsistencies in detection of anticardiolipin antibodies. Am J Reprod Immunol Microbiol. 1989; 19: 78.

25. Scott, J.R., Rote, N.S., Branch, D.W. Immunologic aspects of recurrent abortion and fetal death. Obstet Gynecol. 1987; 70: 645.

26. Branch, D.W., Hales, K., Rote, N.S., Dostal,D.A.,Edwin, S. Incidence antiphospholipid antibodies in pregnancy. Am J Reprod Immunol Microbiol, 1987; 14: 1 .

27. Cowchock, S., DeHoratius, R.J., Wapner, R.J., Jackson, L.G. Subclinical autoimune disease and unexplained abortion. Am J Obstet Gynecol. 1984; 152: 367.

28. Khamashta, M.A., Hams, E.N., liharavi, A.E., et al. Immune mediated mechanism for thrombosis: antiphospholipid antibody binding to platelet membrane. Ann Rheum Dis. 1988; 47: 849.

29. Beer, A.E., Kwak,J.Y.H. Recurrent spontaneous abortions immunological evaluation. In: Current theraphy in obstetrics and gynecology. Philadelphia: Saunders. 1993.

30. Branch, W., Scott, J.R., Kochenour, N.K., Hershgold, E. Obstetric complications associated with the lupus anticoagulant. N Engl J Med 1985; 313: 1322-1326.

31. Billingham, R.E., Beer, A.E. Reproductive Immunology: past, present and future. Perspect Biol Med 1984; 27: 259-275.

32. Bahar, A.M., Kwak JYH, Beer, A.E. Antibodies to phospholipids and nuclear antigens in non-pregnant women with unexplained spontaneous recurrent abortions. J Reprod Immunol. 1993.

33. Grossman, C.J. Regulation of the immune system by sex steroids. Endocr. Rev. 5: 1984; 435-455.

34. Lockwood, C.J., Reece, E.A., Romero, R. and Hobbins, J.C. Antiphospholipid antibody and pregnancy wastage. Lancet, 1986; 11: 742-743.

35. Fish, B., Rikover, Y., Shohat, L., Zurgil, N., Tadir, Y., Ovadia, J., Wik, I, and Yron, I. The relationship between in vitro fertilization and naturally occuring antibodies: evidence for increased production of antiphospholipid antibodies. Fertil Steril. 1991; 56(4): 718-724.

36. Steptoe and Edwards. Birth after the reimplantation of a Human Embryo. Lancet 1978; (2): 366. 